Semiconductor technology

\title{
Kinetics of pulsed laser annealing
}

from Ian W. Boyd and Steven C. Moss

PULSED laser annealing (PLA) is a technique which uses intense bursts of laser radiation to repair the damage produced by ion implantation of crystalline semiconductors, thus improving the structural order and the electrical properties of the annealed material. The mechanism behind the technique has been the subject of a great deal of heated debate over the past few years ${ }^{1,2}$, but evidence compiled from a wide variety of measurements now seems to favour strongly a thermal model over a non-thermal model.

Initially, microscopic examination of the irradiated surface of the semiconductor and evidence for the redistribution of impurity atoms within the material suggested that it had melted and then recrystallized by liquid-phase epitaxial regrowth. In that case, the laser radiation was thought to excite electrons in the valence band across the bandgap and into the conduction band, from which they very rapidly relaxed back to the valence band, transferring their energy to the lattice, and thereby heating up and melting the material. Alternatively, it was suggested that so many electrons could be excited into the conduction band that the usually strong covalent bonding of the solid would be severely weakened. In that case, it was possible to envisage a phase transition whereby, instead of violent atomic vibrations breaking the solid into a hot liquid phase in the usual thermal melting sense, the material 'wobbled' itself gently into a cool jelly-like structure.

There followed a period of unprecedented activity in numerous laboratories to differentiate between the two models. Unfortunately, many experiments were performed too hastily, some were extremely difficult, and others were extremely difficult to interpret. Early results favoured the non-thermal model, which was vague enough to accommodate many strange phenomena. However, over the following years, experiments were repeated, reanalysed and sometimes reinterpreted. On the basis of experiments centred around the measurement of the lattice temperature during and after the annealing pulse, an onslaught on the nonthermal model was mounted. The structure and physical properties of the irradiated material were also subjected to unique and most ingenious investigations ${ }^{1,2}$.

Early support for the thermal model was strongly initiated by time-resolved reflectivity measurements on a nanosecond scale, using a weak probe beam centred on the irradiated layer during the annealing event ${ }^{3}$. The observed high reflectivity phase was characteristic of liquid silicon and transient transmission measurements in the same regime have recently been shown to be consistent with melting ${ }^{4}$. Previous measurements of the same kind, which had appeared to favour non-thermal mechanisms, were apparently in error, because of detector artefacts. Time-offlight mass spectrometry, in conjunction with transient reflectivity ${ }^{5}$, enabled an analysis of the atoms ejected from the surface during the annealing process. Early measurements provided some support for the non-thermal model by showing that melting occurred only for pulses more than 75 per cent greater in energy than the previously determined annealing threshold, but a careful repeat of the experiment has since shown that the evaporation rate at the PLA threshold is indicative of temperatures exceeding the melting point ${ }^{5}$. Independent photoemission studies (see $\mathbf{R}$. T. Williams et al. in ref. 2), as well as timeresolved structural measurements with synchrotron $\mathrm{X}$ rays ${ }^{6}$ and low energy electron diffraction 7 , are also in agreement with the thermal-melting model. Transient electrical conductance of the irradiated material not only yields strong evidence of rapid melting but also provides quantitative estimates for melting and solidification velocities ${ }^{8}$.

Great controversy has surrounded the interpretation of several sets of Raman scattering data that have been recorded at particular frequencies during the laser annealing cycle. The earlier work was seen as strong evidence against thermal melting, since maximum lattice temperatures around $600 \mathrm{~K}$ were deduced (silicon melts at $1685 \mathrm{~K}$ ). More recently, frequencyresolved Raman data taken at fluences just below the transition threshold (the high reflectivity phase cannot be Raman active) have indicated that temperatures close to the melting temperature of silicon can be reached. Furthermore, temporal resolution artefacts during the kind of high energy PLA used in earlier measurements result in misleadingly low estimates of the maximum temperature (see Von der Linde et al. in ref. 1).

Clearly, in the nanosecond excitation regime, high lattice temperatures and melting occur in extremely short time periods. But how long does it take for the energy in the highly excited electronic plasma to be transferred to the lattice? Picosecond studies of laser-induced phase transitions have been most useful in elucidating the various optical- and electronicscattering phenomena. Using $15-20$ ps pulses, and monitoring the reflectivity and transmission of a weak probe beam, it has been deduced that lattice melting occurs during the pulse duration (ref. 9, and Bucksbaum \& Baker in ref. 1) and that the maximum electron-hole plasma concent- ration during the 20 ps pulses was less than $10^{21} \mathrm{~cm}^{-3}$ (ref. 9). Also, by observing the thermally-induced variation of the multiple reflections in silicon-on-sapphire during annealing, direct measurement of lattice heating has been possible (see Lompre et al. in ref. 1). An analysis of the emission of charged particles from the sample surface is also consistent with thermal melting, sets an upper limit of $5000 \mathrm{~K}$ on the electronic temperature and suggests that thermalization between carriers and lattice occurs within $10 \mathrm{ps}$ (see Malvezzi et al. in ref 1).

In the course of rapid melting, it is possible to obtain such fast cooling rates that the liquid layer freezes in an amorphous configuration before recrystallization can occur ${ }^{10}$. Such 'amorphization' is clearly consistent with thermal arguments. Optically-induced phase transitions have also been studied during the past year using 55-90 fs pulses. Time-resolved reflectivity clearly shows that the energy transfer from the excited carriers to the crystal lattice, and subsequent melting, occurs within a few picoseconds ${ }^{11}$. An independent study of the structural changes, in which the symmetry dependence of the secondharmonic generation from a weak probe pulse reflected from the crystal was monitored, clearly indicated a transition from order to disorder on a time scale less than a picosecond, again consistent with rapid melting ${ }^{12}$. However, an analysis of recent measurements of the self-induced reflectivity of intense $100 \mathrm{fs}$ pulses incident upon silicon requires carrier densities very close to the total number of valence electrons, and energy relaxation rates that are 300 times larger than the low density relaxation rates ${ }^{13}$. If confirmed, these results may once again imply the necessity of a nonthermal role in the kinetics of laser-induced 'melting' on the femtosecond time scale. On this time scale, however, the photoexcited system is far from equilibrium, and the definitions applicable to longer time scales become less appropriate to describe any of the transient states of the matter. Until the appropriate measurements are performed and until physical properties are redefined in this regime, the now well worn and metamorphic non-thermal model must remain on the desks of the inventors.

1. Energy Beam-Solid Interactions \& Transient Thermal Processing (eds Fan, J.C.C. \& Johnson, N.M., North Holland, 1984)

2. Laser and Electron-Beam Interactions with Solids (eds Appleton, B.R., \& Celler, G.K., North-Holland, 1982).

3. Auston, D.H., et al. Appl. Phys. Lett. 33, 437 (1978).

4. Lowndes, D.H. Phys. Rev. Letl. 48, 267 (1982)

4. Lowndes, D.H. Phys. Rev. Lett. 48, 267 (1982).

S. Pospieszczyk, A. el al. J. appl. Phys. 54, 3176 (1983).

6. Larsen, B.C. et al. Appl. Phys. Lett. 42, 282 (1983).

7. Becker, R.S. et al. Phys. Rev. Lett. 52, 307 (1984).

8. Galvin, G.J. et al. Phys. Rev. Lett. 48, 33 (1982).

9. Liu, J.M. et al. Appl. Phys. Lett. 41, 643 (1982).

0. Boyd, 1.W. et ai. Appl. Phys. Lett. 45, 80 (1984).

1. Shank, C.V. et al. Phys. Rev. Letl. 50, 454 (1983).

12. Shank CV et al Phys. Rev. Lett 51, 900 (1983).

Ian W. Boyd is in the Department of Electronic and Electrical Engineering, University College, London WCIE 6BT, UK: Steven C. Moss is at Aerospace Corporation, PO Box 92957, Los Angeles, California 90009, USA 